\title{
ARTICLE
}

\section{Experimental validation of a MCNP-based model to predict portal dose images from MLC beams}

\author{
Belén Juste $^{\mathrm{a}^{*}}$, Rafael Miró ${ }^{\mathrm{a}}$, Daniel Morera ${ }^{\mathrm{a}}$, Gumersindo Verdúa ${ }^{\mathrm{a}}$ Juan Manuel Campayo ${ }^{\mathrm{b}}$ and Sergio Díez ${ }^{\mathrm{b}}$ \\ ${ }^{a}$ The Institute for Industrial, Radiophysical and Environmental Safety (ISIRYM), Polytechnic University of Valencia. Camí de Vera, \\ s/n 46022, Valencia , Spain; ${ }^{b}$ Hospital Clínic Universitari de València, Avda. Blasco Ibáñez, 17. Valencia. 46010, Spain
}

\begin{abstract}
This work presents a technique to perform an electronic portal imaging device (EPID) signal planar calibration into dose terms using Monte Carlo simulations. To that, a complete Monte Carlo (MC) simulation of a radiotherapy treatment linear accelerator including a multi-leaf collimator system (MLC) and an EPID has been developed. Several solid water blocks were used to obtain calibration measurements and simulations with a large dose range in order to study the relation between the squared EPID response and the MC calculated dose. The calibrated EPID has also been used as a dosimetric tool to validate the MC MLC Linac model. Simulation and measurement comparisons are below 5\% root mean square of percentage dose difference. This technique could potentially provide a more accurate verification of dose delivery to heterogeneous anatomical regions in patients receiving complex multi-field conformal radiation therapy treatments.
\end{abstract}

Keywords: Monte Carlo; EPID; radiotherapy; $M L C$

\section{Introduction}

Conformal radiotherapy and intensity-modulated radiotherapy (IMRT) techniques involve complex field shaping using multi-leaf collimators (MLCs) and they are increasingly used to treat complex shaped tumors. The accuracy of MLC leaf positioning is essential and the precision of the measurement techniques used for quality control and calibration of these devices is also decisive.

Amorphous silicon electronic portal imaging devices (a-Si EPIDs) were originally developed for patient setup verification. Nowadays, they are increasingly used as dosimeters for IMRT verification and linac quality assurance.

The EPID studied in this work consist on an amorphous silicon array detector which is specially suited for patient positioning verification. We show in this paper the advantages of using this EPID as an alternative to conventional dose monitoring techniques (such as gafchcromic films, diodes and thermoluminescent dosimetry) to verify dosimetric treatment. To that, we have developed a full MC approach which provides an accurate method for dose calculation in specific MLC clinical treatments. In this study we present the comparison between experimental measurements and a MC model of the Elekta Precise radiotherapy facility involving a linac with MLCs and an EPID [1,2].

Previous published works validated the $\mathrm{MC}$ radiation

*Corresponding author. Email: bejusvi@iqn.upv.es therapy unit model using water phantom depth dose curves [6] and forward works included the MLC detailed system in the complete simulation [7], obtaining in both cases excellent results.

\section{Materials and methods}

\subsection{EPID images acquisition}

All the measurements and images acquisitions were performed with an Elekta Sli Precise linear accelerator available at the Hospital Clínic Universitari de València.

The iView GT-type EPID (Elekta) [3] is based on the amorphous silicon detector panel XRD 1640 (Perkin-Elmer Optoelectronics, Fremont, CA) with a fixed source detector distance (SDD) of $160 \mathrm{~cm}$ and a detection area of $46 \mathrm{~cm} \times 46 \mathrm{~cm}$. This system has a 1024 $\mathrm{x} 1024$ pixel resolution and is composed first by a metal layer (Copper and Aluminum) as additional build-up material, in order to maximize deposited dose at the second layer constituted by the scintillator.

Figure 1 shows the Elekta Precise unit radiation head irradiation a solid water slab placed at the treatment table and the EPID flat panel. The experimental set-up presented in Figure 1 involves the acquisition of images maintaining the gantry angle at $0^{\circ}$ and using an square open field size of $20 \mathrm{~cm} \times 20 \mathrm{~cm}$ at the isocentre, and followed by several images of different solid water blocks (from $2 \mathrm{~cm}$ thickness to $20 \mathrm{~cm}$ stepped in $2 \mathrm{~cm}$ increments), with a machine dose rate setting of 100 
monitor units (MU) and a source to isocenter distance (SID) of $100 \mathrm{~cm}$. We have selected these water equivalent blocks thickness in order to obtain a wide grey level intensities range.

Portal images were acquired in the mentioned irradiation conditions using the commercial iViewGT software with a fixed integration time of $433 \mathrm{~ms} /$ frame. All images were generated by integrating the frames acquired during the total radiation dose delivered. The number of frames integrated during beam delivery was estimated to range between 40 and 50 .

Images have been exported from acquisition console to raw format .his and a correction filter map has been applied to each image sequence, in order to eliminate the offset noise, to apply a link offset correction (bad pixels correction) and to perform a heterogeneity detector correction.

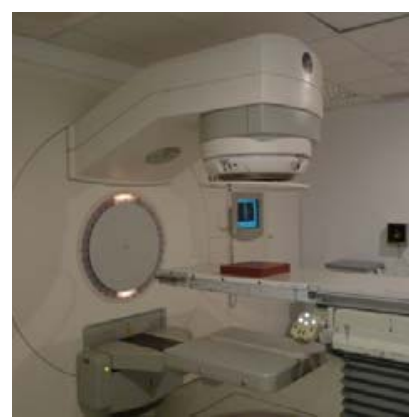

Figure 1. Experimental setup image acquisition.

\subsection{Monte Carlo simulation}

The MCNP version 5 [4] code system has been used to generate an accurate model of the Elekta Precise linear accelerator (operating with a $6 \mathrm{MeV}$ photon beam) incorporating the 80 leaves of MLC and its associated EPID. The detailed geometry of the radiotherapy treatment head unit Elekta Precise, the solid water slabs and the EPID amorphous silicon flat-panel have been accurately implemented in the Monte Carlo model according to the manufacturer data [5].

The validation of MLC MC model was previously validated using depth dose curves in a water phantom [6], [7].

In this work, the response of the imager in the sensitive layer of the detector was simulated in the same irradiation conditions as the experimental procedure was performed.

The MCNP5 code allows to accurately registering the relative electron and photon flux and dose deposition (using the corresponding flux-to-dose conversion factors) at the flat-panel light phosphor layer by means of the FMESH tally.

The pixel resolution of the Monte Carlo EPID model was set to $1 \mathrm{~cm} \mathrm{x} 1 \mathrm{~cm}$ to allow good statistical accuracy in the dose calculation. Since the objective of this work was to perform a first approximation of the possibility of using portal images to obtain dose, it has been chosen this resolution EPID model in order to accelerate calculations, although future works will be addressed towards the improvement of the accuracy of the obtained results, selecting a lower EPID MC resolution.

\subsection{Grey level to dose calibration technique}

The resulting images acquired with the EPID and those obtained by simulation were spatially studied. A regional analysis has been performed in order to map the EPID signal as illustrated in Figure 2. Both the EPID (grey level intensity) and the MC (deposited dose) images for each calibration slab thickness were analyzed using concentric squared sections with $1 \mathrm{~cm}$ separation. In each section, the mean of EPID Signal $\left(\mathrm{S}_{\mathrm{E}}\right)$ and Monte Carlo dose $\left(\mathrm{d}_{\mathrm{MC}}\right)$ was calculated.

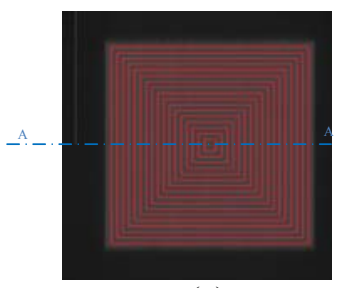

(a)

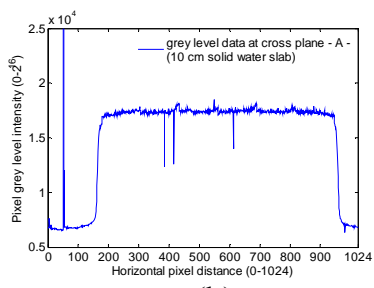

(b)
Figure 2. (a) concentric squared rings used for the analysis of both EPID and MC images and (b) pixel grey level intensity at cross line -A-

An spatial plane calibration matrix was then developed to convert $S_{\mathrm{E}}$ into dose taking into account the dose deposition on-axis and off-axis position.

In this study it has been fixed a 100 MU dose rate, nevertheless, as the response of the EPID is dose rate-dependent, alternatives calibration matrix (using the same methodology) should be perform to each dose rate settings [8].

\subsection{MLC validation}

Once the calibration method described previously was developed, it has also been applied to the verification of the MLC Monte Carlo model of the Elekta Precise.

Our MLC model has been simulated on the basis of technical information provided by the manufacturer and it contains a realistic representation of the leaf design, since the leaf transmission can be an important factor when calculating the patient dose. Since such calculations are very sensitive to the detailed structure of the multileaf collimator, the 80-leaf MCL Elekta Precise were implemented in a geometric developed model, as shown in Figure 3.

Verification measurements in a water phantom were carried out using a Scanditronix Wellhofer chamber for a squared $10 \mathrm{~cm}$ x $10 \mathrm{~cm}$ field MLC shape using a $6 \mathrm{MeV}$ photon beam.

Typical depth dose curves and dose profiles measured with the ion chamber at $10 \mathrm{~cm}$ depth along the horizontal water axis $(\mathrm{SSD}=100 \mathrm{~cm})$ were compared with $\mathrm{MC}$ calculations and are shown in Figures 4 and 5.

The match between computations and measurements (better than 2\%) shows that leaf material, and therefore also transmission properties, were accurately represented. A more complete 2D study has been carried out using the EPID images. In order to verify our MC model further, 

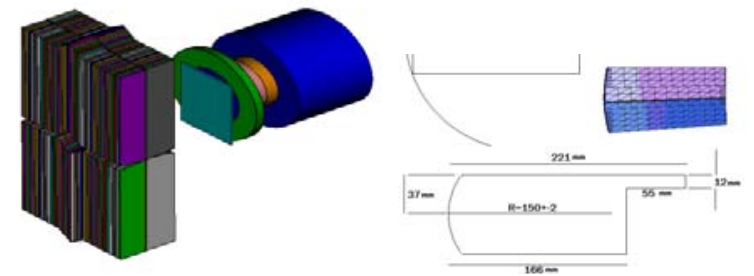

Figure 3. Linac unit head model including the MLC collimator modeled with MCNP5.

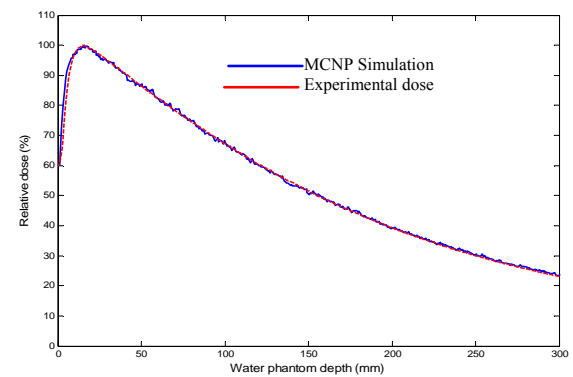

Figure 4. Depth dose curve obtained with a $6 \mathrm{MeV}$ photon spectrum.

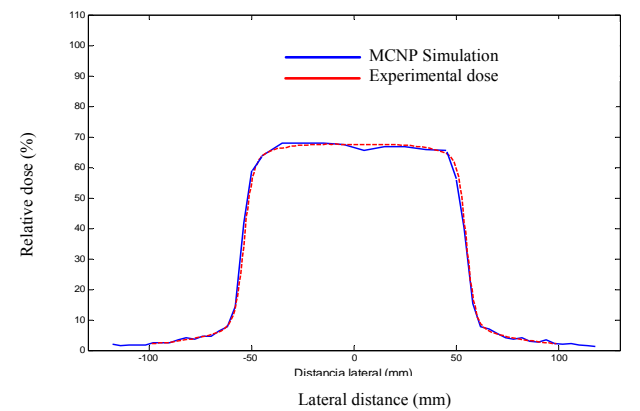

Figure 5. Profile dose at $1 \mathrm{v} \mathrm{cm}$ deptn witn a o $\mathrm{MeV}$ photon spectrum.

several MLC shapes (one rhombus example is shown in this paper) were set up on the linac. The corresponding EPID images were then acquired using the same machine parameters (i.e. $6 \mathrm{MV}, 100 \mathrm{MU}, 160 \mathrm{~cm}$ SDD) as the calibration images. The leaf co-ordinates were then simulated with MCNP MC system.

The resolution of the MC EPID model was set to (1 $\mathrm{cm} \times 1 \mathrm{~cm}$ ) pixel dimension in the detector plane. $\mathrm{S}_{\mathrm{E}}$ was converted to $\mathrm{d}_{\mathrm{MC}}$ using the developed calibration matrix.

\subsection{Results}

\subsubsection{Calibration process}

In this calibration procedure, the EPID signal values $\left(\mathrm{S}_{\mathrm{E}}\right)$ (subtracted the background black image) were normalized to the open field signal. Similarly the MC doses $\left(d_{M C}\right)$ obtained were normalized to the open field simulation dose values. The obtained results demonstrate that there is a linear dependence between EPID signal response and $\mathrm{MC}$ simulated dose.

The comparison between MC results and EPID image is presented. As shown in Figure 6, the different slab thickness (central axis and off-axis) EPID signal $\mathrm{S}_{\mathrm{E}}$ is well represented by a linear function of MC dose $d_{M C}$ and follows the linear relation:

$$
S_{\mathrm{E}}=P \cdot d \mathrm{MC}+O
$$

where $P$ is the $S \mathrm{E} / d_{\mathrm{MC}}$ slope and $O$ is the offset resulting from the linear fit for the central $1 \mathrm{~cm} \mathrm{x} 1 \mathrm{~cm}$ area of figure 2 and the squared ring area at $14 \mathrm{~cm}$ off-axis.

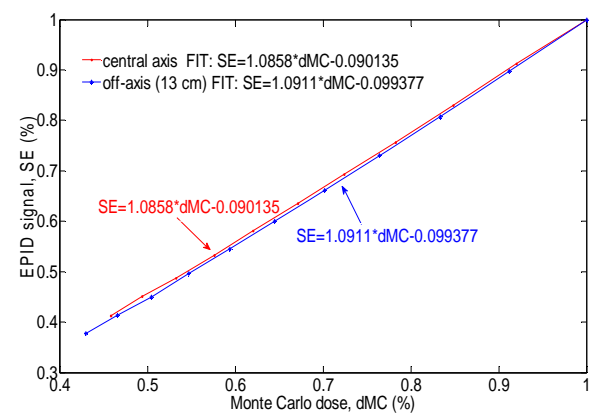

Figure 6. EPID calibration.

For the several off-axis studied, it can be seen that linear relation determine the slope gradients. The calculated linear fit gradient and offset are shown in Figure 7.

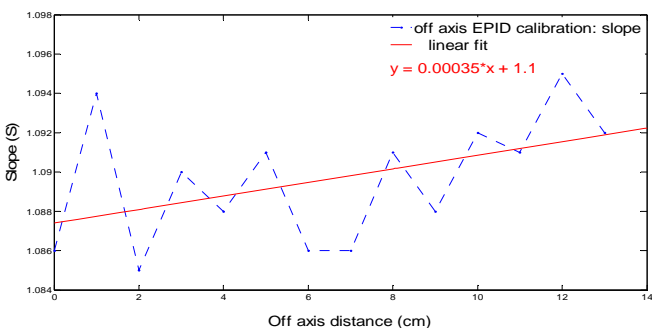

(a)

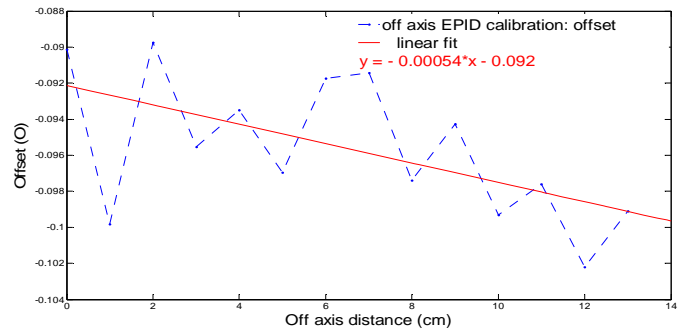

(b)

Figure 7. Off-axis EPID calibration. (a) slopes of the linear fits describing the relationship between SE and dMC. (b) offsets.

As Figure 7 displays, the linear relation is maintained off-axis, but the slope increases with distance from the central axis. Figure 7a represents the slope evolution and its linear function with the off-axis distance for the attenuation curves corresponding to the ith ring of Figure 2. On the other hand, as shown in Figure $7 \mathrm{~b}$ the linear fit offset presents a soft decreasing tendency near zero values.

Using these relations the planar dose delivered to the EPID can be reconstructed from the detector signal $S_{E}$, using the appropriate values $\mathrm{P}$ and $\mathrm{O}$ composing the calibration matrix. This procedure also allows a comprehensive 2D verification of the MLC model. 


\subsubsection{MLC model validation}

To perform an accurate $2 \mathrm{D}$ validation of the MLC MC model, an EPID image of a MLC rhombus shaped field was converted into dose using the calibration matrix described previously. To that, data were processed with MATLAB using a bi-linear interpolation algorithm.

The resulting dose dataset were compared with those obtained by simulation. To that, the complete MCNP5 simulation of the radiation treatment unit head model with the MLC rhombus field was developed. Figure 8 shows the model leaves distribution, and the dose distribution obtained at the flat panel plane using MCNP5.
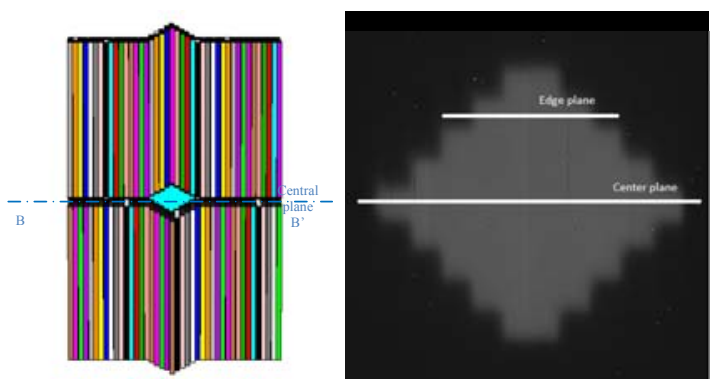

Figure 8. Configuration of the MCNP5 rhombus MLC model and its corresponding image at the EPID plane.

Figure 9 presents that the MC dose profiles analyzed agree accurately with the EPID dose across the whole dose range. The percentage difference between MC dose and calibrated EPID dose is below 5\% root mean square. This shows that the beam segment is accurately simulated and that the actual MLC leaf positions were represented in the MLC simulations with high accuracy degree.

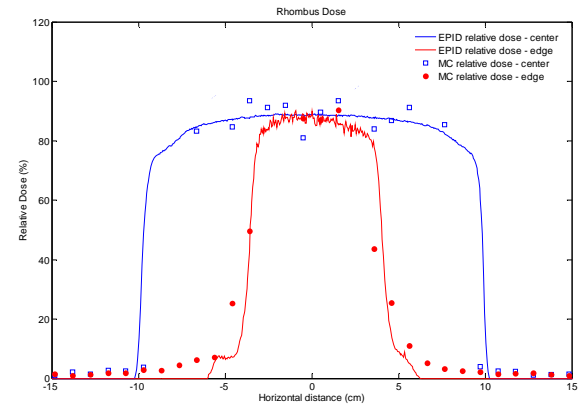

Figure 9. Comparison between MC results and EPID dose.

The excellent agreement proves in this case the validity of the MC model and of the parameters being used, such as leaf-end radius, alloy composition and density.

\section{Conclusion}

MC simulation of electronic portal images can be useful for Conformal Therapy treatment verification. The accurate modeling of geometry, materials, physics particle transport all along radiation unit towards the detector system is a key issue in dosimetric verification of radiotherapy beams, since the accuracy of the results is limited by the model constraints.

Moreover, the calibration of the EPID signal in terms of dose is an essential step in the correct prediction of the dose delivered to the patient.

In this study we have demonstrated a simple method to calibrate EPID images using the MC technique to convert the recorded signal into dose. The simulation and measurement of EPID MLC shaped fields has provided an easy way to develop the physical and geometrical accurate MLC model. Dosimetric comparisons involving a MLC field shape have shown good agreement between simulations and measurements within 5\% root mean square.

The methodology described in this paper has the potential to offer an accurate verification of dose delivery to generally heterogeneous treatment volumes, from complex multi-field or IMRT procedures using devices such as MLCs.

The inclusion of patient (or phantom) $\mathrm{CT}$ is being developed now. MC simulations involving planning CT will allow a better comparison of treatment-time EPID measurements with the reference data to investigate components of uncertainty in dose delivery, such as machine dose output variations, patient displacement relative to intended treatment position and anatomical variations within the patient.

\section{Acknowledgements}

We would like to thank the "Hospital Clínic Universitari de Valencia" and Elekta for the given help.

\section{References}

[1] J.V. Siebers, J.O. Kim, L. Ko, P.J. Keall and R. Mohan, Monte Carlo computation of dosimetric amorphous silicon electronic portal images, Med. Phys., 31(7) (2004), pp. 2135-2146.

[2] B.J.M. Heijmen, K.L. Pasma, M. Kroonwijk, V.G.M. Althof, J.C.J. de Boer, A.G. Visser and H. Huizenga, Portal dose measurement in radiotherapy using an electronic portal imaging device (EPID), Phys. Med. Biol. 40 (1995), pp. 1943-1955.

[3] Elekta iView GT brochure - Elekta Oncolgy Systems.

[4] X-5 Monte Carlo team, MCNP - A General Monte Carlo NParticle Transport Code, Version 5, LA-UR-03-1987, Los Alamos National Laboratory, April 2003.

[5] Information for Monte Carlo Modeling of the iView MV portal imager. Confidential Document $n^{\circ} 4513$ 3710483 03. Elekta Limited.

[6] B. Juste, R. Miró, J.M. Campayo, S. Díez and G. Verdú, Comparison of experimental dose curves in a heterogeneous phantom with results obtained by MCNP5 simulation and treatment planning tools, Applied Radiation and Isotopes 68 (2010), pp. 913-917. ISSN: 0969-8043

[7] B. Juste, R. Miró, J.M. Campayo, S. Díez and G. Verdú, Experimental validation of an accurate multileaf collimation (MLC) Linac simulated by Monte Carlo, Applied Radiation and Isotopes 68 
(2010), p. 4. ISSN: 0969-8043

[8] L. Parent, A.L. Fielding, D.R. Dance, J. Seco and P.M. Evans, Amorphous silicon EPID calibration for dosimetric applications: comparison of a method based on Monte Carlo prediction of response with existing techniques, Phys. Med. Biol. 52 (2007), pp. 3351-3368. 